\section{Revisión de enfoques de políticas alimentarias: entre la seguridad y la soberanía alimentaria (2000-2013)}

\author{
Review of food policy approaches: from food \\ security to food sovereignty (2000-2013)
}

\author{
Revisão de enfoques das políticas alimentares: \\ entre a segurança e a soberania alimentar \\ (2000-2013)
}

\author{
1 Universidad de Antioquia, \\ Medellín, Colombia. \\ Correspondencia \\ L. A. López-Giraldo \\ Universidad de Antioquia. \\ Calle 79 \# 72A-64, Apto. \\ 912, Medellín, Antioquia - \\ 05001000, Colombia. \\ lulopex@gmail.com
}

\begin{abstract}
Food policies have attracted special interest due to the global food crisis in 2008 and promotion of the Millennium Development Goals, leading to approaches by different fields. This thematic review aims to describe the main theoretical and methodological approaches to food security and food sovereignty policies. A search was performed in databases of scientific journals from 2000 to 2013. 320 complete articles were selected from a total of 2,699. After reading the articles to apply the inclusion criteria, 55 items were maintained for analysis. In conclusion, with the predominance of food security as a guiding policy, food sovereignty has emerged as a critical response to be included in designing and researching food policies. Food policies are essential for achieving public health goals. Public health should thus take a leading role in linking and orienting such policies.
\end{abstract}

Nutrition Policy; Food and Nutrition Security; Food Planning
Luis Alirio López-Giraldo 1

Álvaro Franco-Giraldo 1

\section{Resumen}

Las políticas alimentarias han cobrado especial interés, a raíz de la crisis alimentaria mundial de 2008 y el impulso de los Objetivos de Desarrollo del Milenio; dando lugar a enfoques desde diversas orientaciones disciplinarias. Esta revisión temática tiene como objetivo describir los principales enfoques teóricos y metodológicos de políticas, elaborados desde las perspectivas de la seguridad alimentaria y soberanía alimentaria. Se realizó una búsqueda en bases de datos para publicaciones académicas entre 2000 y 2013. De 2.699 publicaciones, se seleccionaron 320 artículos completos. Luego de su lectura para aplicar los criterios de inclusión, se conservaron 55 artículos para el análisis. Se concluye que, frente al predominio de la seguridad alimentaria como orientadora de políticas, emerge la respuesta crítica de la soberanía alimentaria, que debe ser incluida en el diseño y estudio de políticas alimentarias. Éstas son un asunto esencial para el logro de los objetivos de la salud pública. Por tanto, ella debe asumir un rol protagónico, orientador y articulador en dichas políticas.

Política Nutricional; Seguridad Alimentaria y Nutricional; Planificación Alimentaria 


\section{Introducción}

Desde la Asamblea del Milenio de Naciones Unidas (2000) el tema del hambre en el mundo se ubica en un primer lugar en la agenda política internacional y en la de los Objetivos de Desarrollo del Milenio (ODM). Se planteó el desarrollo y la erradicación de la pobreza 1, entendiéndola como "reducir a la mitad el porcentaje de las personas que viven con menos de un dólar diario y a la mitad las personas que padecen hambre"; sin embargo, el enfrentamiento de este problema no ha sido del todo coherente y suficiente como política de los gobiernos. En la última década, el diseño de las políticas públicas alimentarias ha estado orientado por dos perspectivas: la de la Seguridad Alimentaria, emanada principalmente de la visión de los Organismos Multilaterales (OM) y la de la soberanía alimentaria, surgida de las iniciativas críticas de grupos de instituciones de la sociedad civil 2. Este artículo explora la producción académica, referente a las políticas alimentarias, siguiendo estas perspectivas, entre los años 2000 y 2013. Su objetivo es presentar los enfoques teóricos-metodológicos y las estrategias, seguidas en el análisis, la formulación y la implementación de políticas de seguridad y/o soberanía alimentaria (PPS/SA) y su relación con la salud.

Ambas perspectivas deben ser puestas en contexto, ya que en cada país o subregión las problemáticas adquieren características particulares. Así, el examen contextual de las publicaciones desarrolladas en diversos espacios geográficos resulta importante para comprender la complejidad del estudio y el desarrollo de políticas alimentarias. Este trabajo enfatiza la evolución y las posibilidades de desarrollo de la perspectiva política de la soberanía alimentaria, que adquiere cada vez más interés en ámbitos académi$\cos { }^{3}$. Ello, en contraposición a las tendencias predominantes basadas en la seguridad alimentaria de OM 4,5. Además, presenta una revisión de las políticas de alimentación con relación a la salud, expresando la necesidad de reconfigurar la política, teniendo en cuenta las problemáticas de salud pública.

En la última década, entidades como el Instituto Internacional de Investigación sobre Políticas Alimentarias (IFPRI) 6 han reconocido la importancia de las políticas alimentarias internacionales. No obstante, el carácter multidisciplinar del panorama de enfoques hallados en esta revisión muestra claramente un predominio de las interpretaciones desde la economía y la preocupación por los efectos de la globalización económica.

La irrupción del concepto de la soberanía alimentaria en el ámbito político, que ha sido im- pulsado por los movimientos sociales como una respuesta crítica a las limitaciones del concepto de seguridad alimentaria, en cuanto abordaje de los efectos adversos del sistema alimentario globalizado en las economías locales rurales, la pérdida de la agrobiodiversidad, las amenazas al patrimonio agrícola y las consecuencias ambientales de la agroindustria, entre otros. Además del concepto de seguridad alimentaria de la Organización de las Naciones Unidas para la Alimentación y la Agricultura (FAO), que hace referencia al acceso físico y económico a suficientes alimentos inocuos y nutritivos, y del concepto de soberanía alimentaria del Foro de las ONG/ OSC para la Soberanía Alimentaria de 2002 7, que enfatiza en el derecho de los pueblos, comunidades y países a definir sus propias políticas agrícolas, pesqueras, alimentarias y de tierra, existe el concepto de seguridad alimentaria y nutricional. En Brasil, la Ley no 11.346/2006 8 lo define como "la realización del derecho de todos al acceso regular y permanente a alimentos de calidad, en cantidad suficiente, sin comprometer el acceso a otras necesidades esenciales, teniendo como base prácticas alimenticias promotoras de la salud, que respeten la diversidad cultural y que sean ambiental, cultural, económica y socialmente sostenibles". Esta definición de la legislación brasileña avanza hacia una concepción política de la alimentación más integral al tener en cuenta los derechos, la promoción de la salud, la diversidad cultural y el medio ambiente en un entorno de sostenibilidad.

\section{Metodología}

Para la estrategia de búsqueda y la organización de la información, en español e inglés, se utilizaron los descriptores: seguridad alimentaria (food security) AND política (policy), soberanía alimentaria (food sovereignty) AND política (policy) y política alimentaria (food policy). Se seleccionaron publicaciones con los descriptores en el título y en el resumen, en las bases de datos: EBSCO, PubMed, SciELO y Science Direct. La búsqueda se realizó para trabajos publicados en el período 2000-2013.

Los criterios de inclusión se basaron en la exposición de enfoques con al menos dos de los siguientes elementos discursivos: (a) exposición de problemáticas de políticas alimentarias desde las perspectivas de la seguridad o soberanía alimentarias; (b) desarrollo de marcos conceptuales o referentes teóricos; (c) análisis o propuestas metodológicas de políticas alimentarias; (d) evaluaciones de políticas de alimentación; y (e) contextualizaciones sobre políticas alimentarias 
globales. Quedaron excluidas las publicaciones que carecieran de estos elementos.

También se consideró literatura proveniente de OM y Organizaciones No Gubernamentales (ONG). Se visitaron sus páginas web y se realizó una exploración interna de los documentos disponibles, aplicando los mismos criterios de inclusión para las publicaciones académicas, optando sólo por los documentos con directrices para el diseño de políticas alimentarias. Se eligieron los principales organismos alrededor del mundo, interesados en las problemáticas alimentarias de los países en desarrollo, que presentaran enfoques pertinentes para el análisis de políticas alimentarias, con un alcance transnacional.

El análisis se efectuó por dos revisores, uno de ellos con calidad de experto en el tema de la alimentación y ambos con conocimientos en el área de las políticas públicas. Los puntos de discrepancia surgidos entre los revisores fueron resueltos a partir de la búsqueda adicional de literatura científica que permitiera tomar decisiones sobre los artículos más relevantes. Para la extracción de la información relevante se efectuó una clasificación preliminar de los artículos con información sobre problemáticas y políticas alimentarias, desde los enfoques de la seguridad alimentaria y/o la soberanía alimentaria. Se hizo una revisión interna de los artículos para identificar categorías temáticas, luego de obtener la clasificación temática se aplicó ésta en los diferentes artículos y así abstraer la información más pertinente para cada enfoque. Posteriormente, se identificaron y extrajeron citas de los artículos para construir una matriz que permitiera la organización de la información relevante y obtener un panorama de los enfoques mejor sustentados por los autores.

Se identificaron publicaciones que contenían los descriptores en el resumen y, al hacer la lectura del mismo, se cotejó con las que tenían los descriptores en el título. Sólo unas pocas expo- nían enfoques solamente en el resumen y fueron consideradas en el estudio. La revisión hizo énfasis en las publicaciones que contienen los descriptores en el título y que exponen diversos enfoques para el análisis de políticas alimentarias. Para construir la argumentación fue necesario extraer información de todas las publicaciones que incluían uno o dos de los descriptores en el resumen, citando sólo las más significativas.

\section{Hallazgos}

En la Tabla 1 se puede observar que, al considerar sólo el título de la publicación, el concepto de política alimentaria es más prevalente en la literatura que el de seguridad alimentaria AND política y el de soberanía alimentaria AND política, marcando una tendencia que históricamente se ha mantenido. Los trabajos con enfoques de seguridad alimentaria tienden a centrarse en perspectivas económicas y administrativas, encaminadas a mejorar la producción y el comercio de alimentos. Mientras tanto, los trabajos basados en la soberanía alimentaria, se centran perspectivas críticas y contrahegemónicas, con una menor producción, pero mostrando un incremento en el último lustro y constituyendo una tendencia en ascenso.

De las 2.699 publicaciones encontradas con los descriptores en el título y en el resumen, luego de aplicar los criterios de inclusión, la revisión se concentró en 320 publicaciones, con los descriptores en el título. De estos documentos, sometidos a una clasificación conceptual mediante una revisión interna, se seleccionaron 55 pertinentes al objetivo planteado. De las publicaciones de los OM y las ONG se revisaron 20 documentos que exponen directrices para políticas alimentarias.

Así, surgen múltiples enfoques y estrategias en el estudio y el desarrollo de políticas alimentarias (Tabla 2). También se identifican varias ten-

Publicaciones con los descriptores en el resumen y en el título, en cuatro bases de datos entre 2000 y 2013

\begin{tabular}{|c|c|c|c|c|c|c|c|c|}
\hline \multirow[t]{2}{*}{ Descriptor } & \multicolumn{2}{|c|}{ EBSCO } & \multicolumn{2}{|c|}{ PubMed } & \multicolumn{2}{|c|}{ SciELO } & \multicolumn{2}{|c|}{ Science Direct } \\
\hline & Resumen & Título & Resumen & Título & Resumen & Título & Resumen & Título \\
\hline Seguridad alimentaria (food security) & 1.111 & 62 & 219 & 7 & 36 & 1 & 327 & 23 \\
\hline \multicolumn{9}{|l|}{ AND política (policy) } \\
\hline Soberanía alimentaria (food & 39 & 4 & 2 & 0 & 1 & 0 & 8 & 1 \\
\hline \multicolumn{9}{|l|}{ sovereignty) AND política (policy) } \\
\hline Política alimentaria (food policy) & 363 & 116 & 145 & 49 & 16 & 1 & 112 & 56 \\
\hline
\end{tabular}


Tabla 2

Enfoques, marcos, teorías y declaraciones de políticas públicas alimentarias, de seguridad alimentaria y de soberanía alimentaria.

\begin{tabular}{|c|c|c|c|}
\hline Referencias & $\begin{array}{l}\text { Año de la } \\
\text { publicación }\end{array}$ & $\begin{array}{l}\text { País de la } \\
\text { publicación }\end{array}$ & $\begin{array}{l}\text { Enfoques, marcos, teorías y declaraciones de políticas de seguridad } \\
\qquad \text { y/o soberanía alimentaria }\end{array}$ \\
\hline Brooks \& Matthews 21 & 2013 & Francia & Enfoque de gestión de riesgos desde las políticas comerciales. (Seguridad alimentaria) \\
\hline $\begin{array}{l}\text { Nova Scotia Nutrition } \\
\text { Council/Atlantic Health } \\
\text { Promotion Research Centre } 29\end{array}$ & 2004 & Canadá & $\begin{array}{l}\text { Enfoque antipobreza, enfoque de sistemas alimentarios sostenibles, enfoque de desarrollo de } \\
\text { capacidades y el enfoque de la justicia social. (Seguridad alimentaria) }\end{array}$ \\
\hline Hefny 23 & 2012 & Egipto & Enfoque soft path que aboga por la participación y la cooperación. (Seguridad alimentaria) \\
\hline Babu \& Rhoe 24 & 2002 & $\begin{array}{l}\text { Estados } \\
\text { Unidos }\end{array}$ & $\begin{array}{l}\text { Marco conceptual sobre cómo los sistemas agroforestales pueden contribuir a la seguridad } \\
\text { nutricional. Marco teórico para el estudio de las interacciones de los sistemas agroforestales } \\
\text { y la seguridad nutricional. (Seguridad nutricional) }\end{array}$ \\
\hline Kopainsky et al. 25 & 2012 & Noruega & $\begin{array}{c}\text { Marco socio-técnico de análisis de políticas, desde un enfoque dinámico. } \\
\text { (Seguridad alimentaria) }\end{array}$ \\
\hline Paveliuc-Olariu 18 & 2013 & Rumanía & $\begin{array}{l}\text { Enfoque integral para desarrollar una política global que garantice la seguridad alimentaria. } \\
\qquad \text { (Seguridad alimentaria) }\end{array}$ \\
\hline Rocha 26 & 2009 & $\begin{array}{l}\text { Reino Unido/ } \\
\text { Estados } \\
\text { Unidos }\end{array}$ & $\begin{array}{l}\text { Examina el desarrollo de las políticas de seguridad alimentaria y nutricional en Brasil, } \\
\text { haciendo referencia a un enfoque multidimensional que requiere la participación de diversos } \\
\text { sectores de la economía, la sociedad y el gobierno. (Seguridad alimentaria y nutricional) }\end{array}$ \\
\hline Marsden 19 & 2012 & $\begin{array}{l}\text { Reino Unido/ } \\
\text { Estados } \\
\text { Unidos }\end{array}$ & $\begin{array}{l}\text { Enfoque eco-económico que involucra los movimientos agro-alimentarios. } \\
\text { (Seguridad alimentaria) }\end{array}$ \\
\hline Cook 20 & 2002 & $\begin{array}{l}\text { Estados } \\
\text { Unidos }\end{array}$ & $\begin{array}{l}\text { Enfoque basado en el manejo de los recursos para disminuir la inseguridad alimentaria. } \\
\qquad \text { (Seguridad alimentaria) }\end{array}$ \\
\hline Flores et al. 22 & 2005 & $\begin{array}{l}\text { Reino Unido/ } \\
\text { Estados } \\
\text { Unidos }\end{array}$ & $\begin{array}{c}\text { Exploran el enfoque de los medios de subsistencia junto al enfoque de la economía } \\
\text { alimentaria o enfoque de la economía del hogar, iniciado por la organización Save the } \\
\text { Children. (Seguridad alimentaria) }\end{array}$ \\
\hline Hansen-Kuhn 14 & 2011 & $\begin{array}{l}\text { Estados } \\
\text { Unidos }\end{array}$ & $\begin{array}{l}\text { Cuestionamiento al enfoque del libre comercio por su amenaza a los medios de vida rurales, } \\
\text { abogando por la reconstrucción de los sistemas alimentarios locales en el Norte y el Sur. } \\
\text { (Seguridad alimentaria) }\end{array}$ \\
\hline Mahony 15 & 2012 & Australia & $\begin{array}{l}\text { Propuesta de red de alianzas de la cadena de suministro global para afrontar la seguridad } \\
\qquad \text { alimentaria mundial. (Seguridad alimentaria) }\end{array}$ \\
\hline Burlandy 27 & 2009 & Brasil & $\begin{array}{l}\text { Enfoque basado en la intersectorialidad e interrelación con la política económica. } \\
\text { (Seguridad alimentaria y nutricional) }\end{array}$ \\
\hline Paulillo \& Almeida 28 & 2011 & Brasil & $\begin{array}{c}\text { Trabajo comparativo que se apoya en la investigación participativa para evaluar los obstáculos } \\
\text { y la eficacia de los programas en las redes locales. (Seguridad alimentaria) }\end{array}$ \\
\hline Beuchelt \& Virchow 40 & 2012 & Países Bajos & $\begin{array}{l}\text { Enfoque que defiende la tesis de que el concepto del derecho a la alimentación adecuada } \\
\text { es más apropiado para garantizar la seguridad alimentaria que el concepto de soberanía } \\
\text { alimentaria, en el marco de políticas nacionales e internacionales. } \\
\text { (Soberanía alimentaria/Seguridad alimentaria) }\end{array}$ \\
\hline Burmeister \& Choi 41 & 2012 & Países Bajos & $\begin{array}{l}\text { Enfoque que orientado hacia la capacidad de los movimientos sociales transnacionales de } \\
\text { influir en la política agroalimentaria nacional. (Soberanía alimentaria) }\end{array}$ \\
\hline Dupraz \& Postolle 42 & 2012 & Francia & $\begin{array}{l}\text { Enfoque que combina un análisis económico histórico con un enfoque político de los } \\
\text { compromisos de soberanía alimentaria y el comercio. (Soberanía alimentaria) }\end{array}$ \\
\hline Arthur 45 & 2012 & Zimbabue & $\begin{array}{l}\text { Enfoque complementario que involucra los principios de la soberanía alimentaria para } \\
\text { garantizar la seguridad alimentaria en los países africanos. (Soberanía alimentaria) }\end{array}$ \\
\hline Lee 46 & 2012 & Reino Unido & $\begin{array}{l}\text { Análisis del discurso público de la seguridad alimentaria orientada por el comercio y la } \\
\text { soberanía alimentaria como concepto político. (Soberanía alimentaria/Seguridad alimentaria) }\end{array}$ \\
\hline Andrée et al. 43 & 2011 & Canadá & $\begin{array}{l}\text { Enfoque de construcción de la soberanía alimentaria desde abajo con los movimientos } \\
\qquad \text { sociales. (Soberanía alimentaria) }\end{array}$ \\
\hline Tomlinson 38 & 2013 & Reino Unido & $\begin{array}{l}\text { Enfoque desde un discurso alternativo en torno a los conceptos de suministro ecológico de } \\
\qquad \begin{array}{l}\text { alimentos, soberanía alimentaria y agroecología. } \\
\text { (Soberanía alimentaria/Seguridad alimentaria) }\end{array}\end{array}$ \\
\hline
\end{tabular}

(continua) 
Tabla 2 (continuación)

\begin{tabular}{|c|c|c|c|}
\hline Referencias & $\begin{array}{l}\text { Año de la } \\
\text { publicación }\end{array}$ & $\begin{array}{c}\text { País de la } \\
\text { publicación }\end{array}$ & $\begin{array}{l}\text { Enfoques, marcos, teorías y declaraciones de políticas de seguridad } \\
\qquad \text { y/o soberanía alimentaria }\end{array}$ \\
\hline Tomlinson 38 & 2013 & Reino Unido & $\begin{array}{l}\text { Enfoque desde un discurso alternativo en torno a los conceptos de suministro ecológico de } \\
\qquad \begin{array}{l}\text { alimentos, soberanía alimentaria y agroecología. } \\
\text { (Soberanía alimentaria/Seguridad alimentaria) }\end{array}\end{array}$ \\
\hline Pimbert 44 & 2009 & Reino Unido & $\begin{array}{l}\text { Enfoque holístico e integral de la política para la soberanía alimentaria, a partir de } \\
\text { la diversidad de sistemas de alimentación autónomos con equidad, justicia social y } \\
\text { sostenibilidad ecológica. (Soberanía alimentaria) }\end{array}$ \\
\hline Coveney 55 & 2003 & Reino Unido & $\begin{array}{l}\text { Explicación de la relación entre salud pública, gestión ambiental y sostenibilidad como una } \\
\text { oportunidad para la participación y la crítica de la política alimentaria. (Salud pública) }\end{array}$ \\
\hline Pinstrup-Andersen 54 & 2006 & Australia & $\begin{array}{l}\text { Enfoque integral de la salud y la política alimentaria en el logro de los objetivos, tanto de } \\
\text { salud, como de desarrollo. (Salud pública) }\end{array}$ \\
\hline Bellows \& Hamm 61 & 2003 & Reino Unido & $\begin{array}{c}\text { Explicación del potencial para mejorar las políticas y prácticas de seguridad alimentaria a } \\
\text { través de la capacidad de la población para definir y exigir cambios y no en la disposición al } \\
\text { cambio de la burocracia. (Seguridad alimentaria/Salud pública) }\end{array}$ \\
\hline Caraher \& Coveney 62 & 2004 & Reino Unido & $\begin{array}{l}\text { Exploración de la salud pública nutricional a través del control global de la salud y las } \\
\text { formas en que la elección de alimentos está moldeada por muchos intereses. (Salud pública } \\
\text { nutricional) }\end{array}$ \\
\hline FAO 48 & 2006 & Italia & $\begin{array}{l}\text { Informe de Políticas } N^{\circ} 2 \text {. Seguridad alimentaria. Enfoque institucional de la FAO, acerca de } \\
\text { la evolución de los conceptos normativos de la seguridad alimentaria. (Seguridad alimentaria) }\end{array}$ \\
\hline IFPRI 7 & 2005 & $\begin{array}{l}\text { Estados } \\
\text { Unidos }\end{array}$ & $\begin{array}{c}\text { Estrategia del IFPRI, basada en los cambios del contexto político y económico. (Seguridad } \\
\text { alimentaria y nutricional) }\end{array}$ \\
\hline $\begin{array}{l}\text { Foro de las ONG/OSC para la } \\
\text { Soberanía Alimentaria } 7\end{array}$ & 2002 & Italia & $\begin{array}{c}\text { Declaración política que define la soberanía alimentaria como el derecho de los países y } \\
\text { los pueblos a definir sus propias políticas agrarias, de empleo, pesqueras, alimentarias y de } \\
\text { tierra. (Soberanía alimentaria) }\end{array}$ \\
\hline
\end{tabular}

FAO: Organización de las Naciones Unidas para la Alimentación y la Agricultura; IFPRI: Instituto Internacional de Investigación sobre Políticas Alimentarias.

dencias y teorías y marcos en política alimentaria: teoría sistémica, gestión de riesgos, un marco socio-técnico de análisis de políticas, el marco teórico de doble enfoque de la FAO, el modelo bioeconómico, el eco-económico y el enfoque del derecho humano a la alimentación, entre otros.

\section{La perspectiva de la seguridad alimentaria en las políticas alimentarias}

Según la FAO, "existe Seguridad Alimentaria cuando todas las personas tienen en todo momento acceso físico, social y económico a suficientes alimentos inocuos y nutritivos para satisfacer sus necesidades alimenticias y sus preferencias en cuanto a los alimentos, a fin de llevar una vida activa y sana" 9. Holt-Giménez \& Patel 10 plantean que la política pública internacional en materia de alimentación se ha estructurado mayoritariamente en la línea que privilegia el desarrollo del comercio internacional. Desde 1995, año en que se creó la Organización Mundial del Comercio (OMC), se agregó a su agenda la agricultura y los derechos de propiedad intelectual, cuyos efectos se interpretan desde posiciones contradictorias. Sus detractores señalan que tal injerencia destruye las pequeñas economías locales agrícolas, con consecuencias graves para la seguridad alimentaria: un sistema privatizado de la seguridad alimentaria internacional, institucionalizado en los protocolos de la OMC 11, mientras que los defensores de la seguridad alimentaria, basada en el libre comercio, plantean que debería ser posible conciliar el objetivo de la liberalización del comercio agrícola internacional con la aplicación de políticas eficaces de seguridad alimentaria en los países en desarrollo 12 .

Las críticas a los enfoques fundamentados en el libre comercio se centran en la amenaza que éste tiene para las pequeñas economías agrícolas locales de subsistencia, orientando su análisis a los denominados regímenes alimentarios 13 para explicar el rol estratégico de la agricultura y la alimentación en el desarrollo de la economía capitalista global. Hansen-Kuhn 14 plantea que la política de seguridad alimentaria debería centrarse en la reconstrucción de los sistemas alimentarios locales en el Norte y el Sur. Su interpretación sostiene que las inversiones para aumentar la producción de los pequeños agricultores locales en el Sur, será socavada por las importaciones de los productos estadounidenses.

En una dirección opuesta, Mahony 15 (p. 501), defendiendo la idea de adquisición de tierras por 
extranjeros en Australia, señala que "para hacer frente al reto de la Seguridad Alimentaria mundial se requiere que el sector emule el éxito de la industria manufacturera global dentro de una red de alianzas de la cadena de suministro global". No obstante, esta estrategia tiene poco que decir acerca de la pérdida de la biodiversidad 16 a causa de los monocultivos, propios del sistema agroalimentario globalizado. Así, la conversión de tierras para la agro-exportación, incuban movimientos de soberanía alimentaria que expresan relaciones alternativas con la tierra, la agricultura y la alimentación 17 .

Como respuesta a las visiones sectorizadas, que enfatizan sólo en uno o varios sectores de la producción de alimentos, surgen enfoques de carácter integral que comienzan a cuestionar la eficacia de los enfoques tradicionales de políticas de seguridad alimentaria, aunque siguen bastante anclados en el asunto de la productividad. Así, el interés de contextualizar las problemáticas de la seguridad alimentaria, en el ámbito de las políticas globales, aparece con mayor fuerza en el último lustro. Paveliuc-Olariu 18 enfatiza en la necesidad de desarrollar una política global para garantizar la seguridad alimentaria. Su enfoque integral plantea aumentar la competitividad de los países en desarrollo, creando un marco de políticas globales con base en las necesidades y las demandas de las partes interesadas. Su análisis recoge opiniones sobre la posibilidad de cambiar los instrumentos actuales de la política para lograr un intercambio coherente de información y buenas prácticas en todo el mundo; relacionando los riesgos ambientales con la vulnerabilidad de la salud humana. Esta perspectiva abre la puerta a enfoques de política más cercanos a la soberanía alimentaria.

Marsden ${ }^{19}$ parte de una crítica al paradigma alimentario dominante al que denomina "bioeconómico", para plantear una modernización ecológica "real" de la agricultura, a la que llama "eco-economía”. Su enfoque eco-económico tiene en cuenta los movimientos agro-alimentarios, utilizando los principios agroecológicos vinculados a la creación de conocimientos locales. En lo político, enfatiza la importancia de la participación y la autosuficiencia de las comunidades por medio de redes agroalimentarias que facilitan la gobernanza regional y el desarrollo de consorcios, enmarcados en el comercio justo. Puede verse, a pesar de la denominación, un giro hacia la soberanía alimentaria. Este enfoque, contrasta con el predominio de enfoques que privilegian el buen manejo de los recursos para disminuir la inseguridad alimentaria. Por ejemplo, Cook 20 (p. 152), en el contexto estadounidense, sostiene que "los recursos para apoyar los programas dependen del flujo y el reflujo de los ciclos presupuestarios anuales de las políticas estatales y federales". Si bien esto es cierto, se requiere tener en cuenta las fuerzas sociales, económicas y políticas que influyen en la problemática de la inseguridad alimentaria.

Brooks \& Matthews ${ }^{21}$ plantean el enfoque de gestión de riesgos desde las políticas comerciales, abogando por la apertura del comercio, acompañada con instrumentos políticos, como una oportunidad para los países en desarrollo de mejorar su seguridad alimentaria. Ello significa que los agricultores de los países pobres tienen más dificultades para gestionar el riesgo de los precios que sus contrapartes en los países desarrollados 21 . Así, se ofrecen alternativas de protección al comercio como la Plataforma de Gestión de Riesgo Agrícola (PARM), impulsada por OM, como el Fondo Internacional de Desarrollo Agrícola (FIDA), la Comisión Europea, el Banco Mundial y la FAO, entre otros.

De otro lado, Flores et al. 22 exploran la seguridad alimentaria en contextos de crisis prolongadas, proponiendo marcos de política más eficaces. Según su estudio, mientras las crisis prolongadas amenazan el acceso de las personas a alimentos suficientes, el compromiso internacional para reforzar la seguridad alimentaria en esos contextos se ha caracterizado por un fracaso de la formulación de políticas de respuesta, acorde con las cambiantes condiciones económicas, sociales y políticas 22 . Sus reflexiones señalan la existencia de una brecha en la política de seguridad alimentaria y la falta de un acuerdo sobre los principios y los enfoques para llevarla a la práctica.

Dado que las intervenciones adoptadas por los OM no han sido suficientes para mejorar la seguridad alimentaria, y que los ODM no se han cumplido en su totalidad, Hefny 23 cuestiona la eficacia de los enfoques tradicionales, defendiendo la participación y el cambio hacia un comportamiento cooperativo, planteando un enfoque de camino suave, para el acceso sostenible al agua y un cambio de conducta como instrumento de política. A la par se da la preocupación por desarrollar sistemas de producción de alimentos sostenibles. Por ejemplo, Babu \& Rhoe 24 proponen un marco conceptual para identificar la forma cómo los sistemas agroforestales pueden contribuir a la seguridad nutricional. Adicionalmente, desarrollan un marco teórico para estudiar las interacciones de los sistemas agroforestales y la seguridad nutricional, identificando las implicaciones políticas y las necesidades de investigación. Este enfoque defiende los sistemas agroforestales, subrayando la necesidad de investigación en políticas para mejorar la eficiencia de los mercados de alimentos. 
Los determinantes de los diversos enfoques van ampliándose a esferas que trascienden el acceso a los alimentos. Con una perspectiva dinámica, Kopainsky et al. 25 desarrollan un marco socio-técnico de análisis de políticas públicas, que tiene por objeto mejorar la comprensión de la transformación de la agricultura de subsistencia y evaluar las políticas de apoyo a esta transformación. Usando un modelo de simulación adoptan determinantes y procesos descritos en la literatura, confirmados por análisis empíricos; cuyos determinantes más importantes son la utilidad local de semillas, la compatibilidad con las prácticas agrícolas de subsistencia y las habilidades para el cultivo, entre otros.

Estos determinantes deben conectarse con la participación para aproximarnos a lo que podría ser un enfoque sistémico. En Brasil, Rocha 26 defiende las que califica de políticas gubernamentales audaces e innovadoras, respaldadas por nuevas formas de participación popular en la política social para la alimentación y la seguridad nutricional. Señala que la esencia de la estrategia Hambre Cero institucionaliza como la responsabilidad del Estado, bajo la comprensión de la seguridad alimentaria y la nutrición como un problema sistémico e integral debe ser abordada desde un enfoque multidimensional, con la participación de diversos sectores de la economía, la sociedad y el gobierno.

La importancia de la intersectorialidad en la interrelación con la política económica es señalada por Burlandy 27 para analizar las estrategias institucionales del gobierno federal de Brasil en la promoción de la intersectorialidad en seguridad alimentaria y nutrición. Burlandy 27 sostiene que la institucionalidad gubernamental no es suficiente para promover la intersectorialidad y que es importante la inducción de procesos. Propone la combinación de diferentes mecanismos institucionales para lograr la intersectorialidad: consejos integrados y presididos por sectores gubernamentales y la sociedad civil; apoyo político desde la presidencia, y la inclusión como una cuestión estratégica en el gobierno, entre otros.

No obstante, tanto la participación como la intersectorialidad plantean interesantes desafíos para el estudio de políticas de seguridad alimentaria. En Brasil, Paulillo \& Almeida 28 describen y comparan las características de las redes públicas de las políticas locales de seguridad alimentaria para identificar barreras institucionales y de organización. Su trabajo comparativo se apoya en la investigación participativa para evaluar los obstáculos y la eficacia de los programas en las redes locales; identificando factores estructurales influyentes como el grado de control central y el nivel de recursos. Las experiencias brasile- ñas que enfatizan la participación y la intersectorialidad revelan la complejidad de las políticas alimentarias, en lo referente a la necesaria confluencia de actores sociales y políticos, muchas veces con intereses diversos, en el desarrollo de estrategias de intervención.

En Canadá, los enfoques se centran en el problema de la inseguridad alimentaria. Según el Atlantic Health Promotion Research Centre (AHPRC) 29, son dos los enfoques que intentan comprender dicho problema: el enfoque antipobreza y el enfoque de sistemas alimentarios sostenibles. El primero ve la pobreza como un problema prioritario subyacente; el segundo, centra su atención en la producción y la venta al menudeo como problemas del sistema alimentario. Según el AHPRC, a pesar de las diferencias entre estos enfoques parece existir consenso en la necesidad de rediseñar las estrategias para generar cambios fundamentales en la forma de entender la alimentación y las causas profundas de la inseguridad alimentaria, a su vez asociada con los determinantes de la salud. El AHPRC sugiere además que la investigación-acción participativa es una oportunidad de crear capacidades e inclusión social en el proceso de formulación de políticas.

\section{La perspectiva de la soberanía alimentaria en las políticas alimentarias}

En 2005 la FAO publica el libro Políticas de Seguridad Alimentaria en los Países de la Comunidad Andina ${ }^{30}$, el cual se convierte en una directriz importante en la materia para las naciones de la región. Luego, aparece la iniciativa de elaboración de un Marco Estratégico Mundial para la Seguridad Alimentaria y la Nutrición (GSF) por el Comité de Seguridad Alimentaria Mundial (CFS) 31 que se materializa en 2012 en un primer proyecto, como una herramienta para la formulación de políticas y la toma de decisiones en materia de seguridad alimentaria y nutrición 32 .

En la literatura proveniente de las ONG se ha desarrollado el concepto de soberanía alimentaria como una respuesta crítica al concepto de seguridad alimentaria. Dicho concepto se introdujo en el debate internacional por la ONG La Vía Campesina en 1996, durante la Cumbre Mundial sobre la Alimentación, como: el "derecho de cada nación para mantener y desarrollar su propia capacidad para producir los alimentos básicos de los pueblos, respetando la diversidad productiva y cultural" 2. En 2000, el concepto se amplía al "derecho de los pueblos a definir su propia política agrícola y alimentaría sin dumping hacia otros países" 33 .

La presión política de estas organizaciones de la sociedad civil ha hecho que los OM comiencen 
a reconocer el asunto de la soberanía alimentaria y, prueba de ello, es la aceptación de la FAO de incluirla en el debate político. Montagut \& Dogliotti 34 abordan la soberanía alimentaria, en relación con la defensa de los derechos de las comunidades campesinas e indígenas afectadas por la globalización económica. Centrada en el propósito de apoyar los mercados y la producción local, esta perspectiva se opone radicalmente tanto a la producción para la exportación de alimentos como a su importación, en consonancia con los enfoques centrados en los derechos humanos y el derecho a la alimentación. Según Windfuhr \& Jonsén 35 , estos son instrumentos jurídicos de referencia que proporcionan estándares legales a las políticas, teniendo en cuenta que se requiere un marco para que los estados cumplan con sus obligaciones, en relación con dichos derechos, que irrumpen con mayor fuerza en épocas de crisis.

Así, un debate central a la raíz de la crisis alimentaria 36 , ligada al régimen alimentario neoliberal 37 y agudizada en 2008, es sobre aumentar o no la producción de alimentos. Tomlinson ${ }^{38}$ critica radicalmente la idea de duplicar la producción mundial para 2050, señalando que tal cuestión nunca se ha pensado como una meta normativa de la política. En el contexto de la crisis, también se critica la liberalización del comercio agrícola internacional, asociada a políticas neoliberales 10 , al tiempo que se fortalecen los movimientos sociales que defienden la soberanía alimentaria y las deliberaciones que ven en ella un requerimiento fundamental de la seguridad alimentaria en el contexto de la globalización 39 .

Beuchelt \&Virchow 40 , al discutir sobre la idoneidad del concepto de soberanía alimentaria para ser utilizado por los hacedores de políticas públicas nacionales e internacionales, se decantan por la utilización de la noción del derecho humano a la alimentación adecuada como la más promisoria. Reconocen que, a pesar de que el concepto de soberanía alimentaria ha hecho camino tanto en los debates y las políticas de los $\mathrm{OM}$, como en los de los gobiernos naciones, el enfoque jurídico del derecho a la alimentación resulta más apropiado para el desarrollo de políticas nacionales e internacionales encaminadas a reducción del hambre, dado que se aplica a todos los seres humanos, incluyendo a los pequeños productores de alimentos.

Por su parte, quienes defienden la fuerza del concepto de la soberanía alimentaria en la elaboración de las políticas, la mantienen como la principal alternativa. En Corea del Sur, Burmeister \& Choi ${ }^{41}$ examinan su alcance e inclusión en la agenda política agroalimentaria. Su análisis se centra en el espacio multinivel (transnacional y nacional) de las políticas, con relación a la abogacía que ejercen las organizaciones transnacionales de la sociedad civil, ante el régimen global del gobierno neoliberal de la OMC.

Este interés por la relación entre lo transnacional y lo nacional en materia de soberanía alimentaria resulta fundamental para el diseño de políticas agroalimentarias. Con el propósito de estudiar si las naciones de África Occidental pueden alcanzar la soberanía alimentaria a la par con sus compromisos comerciales y otras restricciones externas, Dupraz \& Postolle 42 desarrollan un enfoque que combina un análisis económico histórico con un enfoque político de los compromisos de la soberanía alimentaria y el comercio, considerando objetivos y estrategias de políticas.

Andrée et al. 43, al hablar de construir alianzas inusuales, en torno a la soberanía alimentaria en Canadá, defienden la idea de un enfoque "ascendente" para construir soberanía alimentaria en Canadá; haciendo mención al People's Food Policy Project (PFPP), desde el cual de desarrollan procesos de consulta y políticas con líderes del Food Secure Canada (FSC) en asociación con movimientos sociales. Este enfoque parte del interrogante por las posibilidades y los límites de una construcción contrahegemónica en torno a los principios de la soberanía alimentaria. Por ejemplo, se dice que el creciente poder de los consumidores podría hacer contrapeso al creciente poder de la industria. Sin embargo, dicho poder de los consumidores no prioriza los valores públicos, ni las necesidades de los más marginados.

\section{Discusión}

Los enfoques para el análisis de políticas alimentarias difieren en sus contenidos, según la perspectiva la seguridad alimentaria o soberanía alimentaria. En la primera, predominan el aumento de la producción, la gestión del riesgo agrícola, la gerencia de recursos, el libre comercio, los medios de subsistencia a nivel individual, el acceso, la distribución y la sostenibilidad de los sistemas alimentarios. En la segunda, se enfatiza en la producción local, la protección de la biodiversidad, la equidad, la sostenibilidad ambiental, los modelos agroecológicos y la participación de la sociedad civil, entre otros. Se plantea entonces el interrogante: ¿̇cuál de las perspectivas resulta más adecuada para encarar los desafíos de las políticas alimentarias en el contexto de la globalización? Esta discusión intenta dar algunas señales, al respecto.

Pimbert 44 señala la importancia de un enfoque holístico e integral de la política en sobera- 
nía alimentaria, dado que muchos actores están ahora co-optando por el término de soberanía alimentaria para otorgar autosuficiencia a propuestas que él considera aislacionistas, ya que ignoran los intercambios y las complementariedades entre las regiones. Pimbert 44 (p. 5) sostiene que la noción de "soberanía alimentaria" es quizás mejor entendida como un "proceso de transformación que busca recrear el ámbito democrático y regenerar una diversidad de sistemas de alimentación autónomos basados en la equidad, la justicia social y la sostenibilidad ecológica".

Por su parte, Arthur 45 enfatiza que la soberanía alimentaria, se concreta con la participación ciudadana, la reforma agraria, la promoción de los derechos de propiedad para la población local y el acceso de los pequeños agricultores a los mercados locales y regionales. Estos y otros atributos de la soberanía alimentaria tienen su contraparte en la seguridad alimentaria (Figura 1), a algunos de los cuales nos referiremos para dar cuenta de este debate.

\section{De la seguridad a la soberanía alimentaria}

De acuerdo con Windfuhr \& Jonsén 35 las causas principales del hambre y las barreras a la reducción de la pobreza, que son relevantes para el debate de soberanía alimentaria, son de orden nacional e internacional. Las causas de orden nacional se relacionan con la marginación, el acceso a los recursos productivos y la política agraria, la asignación de presupuestos, el empleo rural y la intervención de otras áreas de política como: la atracción de inversiones extranjeras directas, la privatización de servicios primarios, las industrias extractivas y el VIH/SIDA. Estos argumentos, no sólo revelan asuntos estructuralmente complejos del sistema alimentario global, sino que señalan serios desafíos para las políticas alimentarias a escala local, nacional e internacional. Para los cuales, el concepto de seguridad alimentaria comienza a ser insuficiente.

A pesar de que el concepto de seguridad alimentaria no ha sido sustituido por el concepto de soberanía alimentaria, existe la tendencia a que este último gane espacio en el debate político. Según, Beuchelt \& Virchow 40 el concepto de soberanía alimentaria está empezando a encontrar su camino en los debates y las políticas de las organizaciones de las Naciones Unidas y los gobiernos nacionales, tanto en los países en desarrollo, como en los países industrializados. Éstos señalan que el concepto de soberanía alimentaria resulta apropiado para la adecuación de las políticas internacionales de desarrollo, dirigidas a la reducción del hambre y la pobreza, especialmente con relación al derecho humano a una alimentación adecuada.

De acuerdo con Lee 46 , el discurso internacional de política pública agroalimentaria de la seguridad alimentaria ha estado orientado principalmente por el comercio y se ha estandarizado por medio del fomento comercial internacional de bienes y productos alimenticios; además de la obtención de derechos de propiedad privada, que cada vez más adquieren importancia hegemónica. La seguridad alimentaria orientada al comercio se entendería entonces como una visión mal especificada y cada vez más arcaica,

\section{SEGURIDAD ALIMENTARIA}

Modelo de producción industrial

Libre comercio

Discurso dominante

Gestión de riesgos

Alimentos como bienes o recursos

Agroexportación

Hambre consecuencia de la baja producción

Uso de semillas genéticamente modificadas

Organismos multilaterales

Acceso a la tierra vía mercado

Derecho a la alimentación con predominio de

valores individuales

\begin{tabular}{|c|c|}
\hline & SOBERANÍA ALIMENTARIA \\
\hline & Modelo de producción agroecológico \\
\hline & Protección estatal \\
\hline & Discurso contrahegemónico \\
\hline$\downarrow$ & Sostenibilidad ecológica \\
\hline POÍ́TICAS & Alimentos ligados a los derechos humanos \\
\hline ALMENTARIAS & Producción local \\
\hline 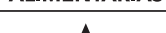 & Hambre consecuencia de la inequidad \\
\hline$\uparrow$ & No uso de semillas genéticamente modificadas \\
\hline & Organizaciones de la sociedad civil \\
\hline & Acceso a la tierra vía reforma agraria \\
\hline & $\begin{array}{l}\text { Derecho a la alimentación con predominio de } \\
\text { valores colectivosedominio de valores individuales }\end{array}$ \\
\hline
\end{tabular}


relativamente indiferente a las sensibilidades ecológicas y limitaciones de recursos. Mientras tanto, la soberanía alimentaria al referirse a los derechos de los pueblos a determinar autónomamente sus políticas agrícolas, va más allá de las políticas de reducción del hambre y la pobreza y el derecho humano a una alimentación adecuada.

Pingali et al. 47 han propuesto un enfoque de doble vía para mejorar la seguridad alimentaria, a través de políticas específicas para situaciones de crisis prolongadas, vinculando intervenciones inmediatas con estrategias a largo plazo de crecimiento sostenible. El doble enfoque de estos autores ha sido tenido en cuenta por la FAO, el cual denomina marco teórico de doble enfoque 48 para combatir el hambre, combinando las preocupaciones de desarrollo agrícola y rural sostenible, con programas específicos para mejorar el acceso directo a los alimentos para los más necesitados. Sin embargo, el predominio de la agricultura industrial se contrapone a la soberanía alimentaria, en el momento de abordar la seguridad alimentaria y el logro de los ODM 49.

Otra tendencia vislumbra enfoques que pugnan por una mirada integral para las políticas alimentarias, que plantean un giro en las políticas y una reestructuración institucional desde el ámbito global hasta el ámbito local. Con lo cual, se deberían integrar ambas perspectivas, seguridad y soberanía alimentaria, desde lo macrosocial (soberanía) a lo micro-social (seguridad). Esto, sin desconocer la afirmación de Gross et al. 50 de que la seguridad alimentaria y nutricional ha evolucionado dramáticamente durante las últimas décadas, en lo referente a su teoría y su práctica, en las cuales se han introducido diversos instrumentos operativos y enfoques conceptuales. Es decir, que la perspectiva nutricional no puede desligarse del contexto social y ambiental para no caer en interpretaciones políticamente irrelevantes 51 .

En otro sentido, Carrasco \& Tejada 52 (p. 14) dicen que no es casual que el concepto de soberanía "surja en el periodo de la globalización neoliberal y la relativa pérdida de autonomía de los estados. Se trata de un concepto transformador, de carácter político y global, que muestra la resistencia a aceptar que los caminos ya están trazados y definidos al margen de la voluntad popular y, en determinadas dimensiones, en contra de los intereses de los pequeños productores, los campesinos, las poblaciones indígenas, etc". Al ser un concepto político y global, la soberanía alimentaria se encuentra fuertemente vinculada con los derechos de participación democrática 53 ; en asuntos como el sistema alimentario, el acceso a la tierra y los métodos de producción, entre otros.

\section{Políticas alimentarias y salud pública}

A pesar de los diversos temas que atraviesan las PPS/SA, nos interesa en esta discusión precisar algunos aspectos de las mismas relacionados con la salud pública. Si bien es evidente la relación entre las políticas alimentarias y la salud de la población, no abundan los trabajos que desarrollen enfoques a profundidad para analizarla. Al estudiar las relaciones de causalidad bidireccional entre el sistema alimentario global, la salud y la nutrición, Pinstrup-Andersen 54 argumenta que tanto la investigación como la política agrícola deben considerar los efectos de dicho sistema en la salud y la nutrición. Este enfoque integral afirma que, en el contexto del sistema alimentario global, se deben tener en cuenta los factores económicos, sociales, culturales y políticos, para lograr objetivos sociales e individuales encaminados a mejorar la nutrición y la salud humana.

Desde una perspectiva sanitaria, Coveney 55 señala que la política alimentaria es un asunto crítico para la salud pública, cuestionando la distancia analítica existente entre la política alimentaria, la salud pública y la promoción de la salud. Su enfoque deja claro que la política alimentaria se encuentra más cerca de la promoción del comercio y la industria que de la promoción de la salud y el bienestar de las poblaciones. Aquí, un factor determinante para la salud pública es la pobreza alimentaria. Según Coveney 55, el asunto clave es preguntarse sobre los intereses que configuraron la política actual, que termina enfatizando la educación de los consumidores para elegir alimentos saludables, pero deja de lado los problemas relacionados con la comercialización y la calidad, entre otros.

Las apreciaciones desarrolladas en esta revisión le apuntan a las políticas alimentarias como determinantes sociales de la alimentación, en primera instancia, pero principalmente de la salud pública y de la salud humana. Un ejemplo, donde resulta notoria la relación entre seguridad alimentaria, salud pública y políticas públicas, se da en la provincia canadiense de la Columbia Británica. Seed 56 afirma que allí la salud pública ha reemergido como conductora de la seguridad alimentaria, integrando políticas, programas e infraestructura al sector sanitario y a otras áreas gubernamentales, incluyendo la seguridad alimentaria como un programa básico de salud pública. Entre los modelos existentes de los determinantes sociales de la salud, el desarrollado en Canadá -en una Conferencia de la Universidad de York- contempla la inseguridad alimentaria como uno de los 14 factores determinantes 57 .

Seed et al. 58 sostienen que en la Columbia Británica las prácticas convergen y divergen 
desde la noción de la seguridad alimentaria comunitaria que ha surgido como un enfoque en Norteamérica, destacando la ambivalencia entre los actores interesados en abordar la inseguridad alimentaria con énfasis en la salud pública como motor de la seguridad alimentaria y los actores de la sociedad civil interesados en la democracia alimentaria. Asimismo, la democratización del sistema agroalimentario tiene que ver con el establecimiento de coaliciones entre diversos grupos de interés 59. En el mismo contexto, y sobre la base de un enfoque centrado en la salud individual y comunitaria, Friendly 60 afirma que para desarrollar una política de seguridad alimentaria se debe promover la vivienda social en las ciudades canadienses.

$\mathrm{Al}$ establecer la discusión sobre el impacto en la salud pública en relación con cómo se define la seguridad alimentaria, Bellows \& Hamm 61 sostienen que los debates sobre la Seguridad Alimentaria a escala global influyen en los actores locales de la salud pública. Lo comunitario no se reduce al desarrollo de programas, sino a la participación de la comunidad en la política de salud pública, en lo referente a la alimentación. Bellows \& Hamm 61 señalan la importancia de la Community Food Security Coalition en el impulso de un marco común de políticas con justicia social, desarrollo sostenible y derechos económicos, como un asunto esencial de salud pública.

Pinstrup-Andersen 54 se centra en las relaciones de causalidad bidireccional del sistema alimentario global con la salud y la nutrición para argumentar que dicho sistema alimentario global comienza y termina en la salud. Por tanto, la priorización de la investigación y la ejecución la política agrícola, en países en desarrollo, deben tener en cuenta sus efectos en la salud y la nutrición. Este autor propende por un enfoque integral de la salud y la política alimentaria que puede ser más eficaz en el logro de objetivos, tanto de salud, como de desarrollo económico, en lugar de las actuales políticas sectoriales. Su enfoque tiene en cuenta asuntos como el acceso al agua potable como un factor importante en la relación entre el medio ambiente y el sistema de alimentación en países pobres; que implica una fuerte relación causal con la salud.

Estos planteamientos llaman la atención sobre el rol y los intereses de los actores del sistema global de alimentación, que no necesariamente se orientan al interés por el bienestar y la salud humana. Caraher \& Covene 62 sostienen que la política alimentaria no ha tratado de forma importante estos asuntos, que inciden en la salud pública nutricional, dado que su enfoque se basa en la nutrición, la elección de los alimentos y la salud biomédica; dejando de lado asuntos ecoló- gicos y de sostenibilidad. Asimismo, Lang et al. 63, abogando por la necesidad de reconfigurar la política, examinan la integración de la salud, el medio ambiente y la sociedad, en el contexto de las políticas alimentarias en países desarrollados; aclarando que la yuxtaposición entre lo local y lo global es central para comprender dicha integración. No obstante, Maceira \& Stechina 64 , en el contexto argentino, llaman la atención sobre la dificultad para abordar el problema alimentario desde un enfoque sanitario. Por tanto, argumentan que la alimentación debe ser considerada como indicador sanitario por los tomadores de decisiones en las intervenciones la salud pública.

\section{Conclusiones}

Aunque la variedad de enfoques de políticas PPS/ SA analizadas en esta revisión, se encuentra dimensionada por el contexto social, económico propio de cada país en el que se implementen, es posible afirmar que predomina la tendencia a implementar enfoques basados en el aumento de la producción de alimentos, el libre comercio, la gestión del riesgo, el asistencialismo, el control de la pobreza y la preocupación por el suministro de alimentos (seguridad alimentaria). Asimismo, en la literatura académica y en los documentos de OM predomina el concepto de Seguridad Alimentaria como orientador de políticas públicas, mientras que el concepto de soberanía alimentaria emerge principalmente en las publicaciones de las ONG como una repuesta crítica, comprometida con la defensa de la producción local de alimentos y el derecho de los pueblos a la autosuficiencia alimentaria.

La necesidad de implementar enfoques integrales para el diseño y la investigación en PPS/SA es cada vez más evidente en el contexto de la globalización, en donde las acciones corporativas y las decisiones políticas sobrepasan los ámbitos nacionales. Con ello, los enfoques de la soberanía alimentaria, que enfatizan en la importancia de la participación, la autosuficiencia de las comunidades y el fortalecimiento de la gobernanza regional y local, aportan elementos importantes al debate sobre la formulación de políticas alimentarias en el ámbito global. En este sentido, la síntesis interpretativa de esta revisión temática muestra la escasa producción académica en torno a la soberanía alimentaria, frente a la vasta producción en torno a la seguridad alimentaria, lo cual, revela a su vez, la necesidad de involucrar la soberanía alimentaria como un tema de interés prioritario, no sólo para la investigación académica y el debate político global, sino para el 
diseño y el estudio de políticas relacionadas con la alimentación.

Con la crisis alimentaria aún por resolver, los enfoques que enfatizan en los derechos humanos, entre ellos el derecho a la alimentación, podrían irrumpir con mayor fuerza si dicha crisis continúa sin resolver. Dichos derechos, constituyen un requerimiento fundamental para afrontar las problemáticas alimentarias asociadas a la globalización. Así, las políticas alimentarias globales se encontrarán con el discurso de la soberanía alimentaria. Si bien los enfoques descritos muestran un abigarrado panorama, sin duda la perspectiva de la soberanía alimentaria es más amplia. En el centro del debate sobre globalización es recurrente el tema de la política internacional y aparecen temas cruciales más allá de la alimentación; la autonomía, el comercio justo, la soberanía nacional, el problema de hambre, la actitud frente a los derechos humanos, la autodeterminación, la defensa de la biodiversidad, los enfoques participativos de políticas alimentarias y la salud pública. Mientras la problemática del hambre requiere la inclusión de estos temas en la agenda de los OM, continúan predominado las estrategias de la seguridad alimentaria orientadas por el comercio.

\section{Resumo}

As políticas alimentares têm alcançado especial interesse por causa da crise alimentar mundial de 2008 e do impulso dos Objetivos de Desenvolvimento do Milênio, dando origem a enfoques de diversas orientações disciplinares. Esta revisão temática tem como objetivo descrever os principais enfoques teóricos e metodológicos de políticas, sob a perspectiva da segurança alimentar e da soberania alimentar. Foi feita uma pesquisa em bases de dados científicas entre 2000 e 2013, num total de 2.699 publicações, das quais foram selecionados 320 artigos na íntegra. Atendidos os critérios de inclusão, foram escolhidos 55 artigos para análise.
Existe debate sobre cuál de los enfoques resulta más apropiado para el diseño de políticas alimentarias o, incluso, si es el enfoque jurídico del derecho a la alimentación el más adecuado. No obstante, no se puede desconocer la amplia gama de posibilidades analíticas y la complejidad interpretativa que aportan los enfoques integrales de la soberanía alimentaria. A pesar de que la literatura académica aún no aborda con profundidad la conexión entre salud pública y soberanía alimentaria en el campo de las políticas públicas alimentarias, es posible afirmar que la soberanía alimentaria constituye una parte fundamental del entramado de la determinación social de la salud, que debería ir de la mano con las estrategias de promoción de la salud y del desarrollo social. Una sinergia pendiente por explorar. Finalmente, esta revisión permitió establecer que existe distancia entre las políticas alimentarias y la salud pública; lo cual se ve reflejado en los escasos trabajos que abordan la relación entre ellas. Las políticas alimentarias son un asunto esencial para la salud pública. Por tanto, consideramos que las PPS/SA deben ser componentes básicos de la salud pública y, los objetivos de ésta deben articular y orientar las políticas alimentarias.
Ao final dessa análise, concluiu-se que, diante do predomínio da segurança alimentar como orientadora de políticas, surge a pergunta crítica pela soberania alimentar, que deve ser incluída na formulação das políticas alimentares e seu contínuo estudo. Trata-se de assunto essencial para alcançar os objetivos da saúde pública e, em consequência, assumir o papel protagonista, orientador e articulador em tais políticas.

Política Nutricional; Segurança Alimentare Nutricional; Planejamento Alimentar 


\section{Colaboradores}

L. A. López-Giraldo contribuyó con la búsqueda de la información en bases de datos, selección de las publicaciones relevantes, análisis de la información y redacción del artículo. Á. Franco-Giraldo contribuyó con el análisis de la información y redacción del artículo.

\section{Agradecimientos}

Esta publicación es resultado de investigación realizada con el apoyo financiero de la Estrategia de Sostenibilidad 2013-2014 del Comité para el Desarrollo de la Investigación (CODI), Universidad de Antioquia.

\section{Referencias}

1. Naciones Unidas. Declaración del Milenio. Resolución 55/2, aprobada por la Asamblea General. New York: Naciones Unidas; 2013.

2. La Vía Campesina. Position of the Vía Campesina on Food Sovereignty. The right to produce and access to land. Roma: World Food Summit; 1996.

3. Program in Agrarian Studies, Yale University; Journal of Peasant Studies. Conference "Food Sovereignty: a critical dialogue". http://www.yale.edu/ agrarianstudies/foodsovereignty/.

4. United Nations. High-Level Task Force on the Global Food Security Crisis. Comprehensive framework for action. New York: United Nations; 2008.

5. Naciones Unidas. Equipo de Tareas de Alto Nivel sobre la Crisis Mundial de la Seguridad Alimentaria (HLTF). Marco amplio para la acción actualizado. Roma: Naciones Unidas; 2010.

6. Instituto Internacional de Investigación sobre Políticas Alimentarias. Estrategia del IFPRI: hacia la seguridad alimentaria y nutricional, investigación sobre políticas alimentarias, fortalecimiento de la capacidad y comunicación, actualización. Washington DC: Instituto Internacional de Investigación sobre Políticas Alimentarias; 2005.

7. Foro de las ONG/OSC para la Soberanía Alimentaria. Soberanía alimentaria: un derecho para todos. Declaración política del Foro de las ONG/OSC para la Soberanía Alimentaria. Roma: Foro de las ONG/OSC para la Soberanía Alimentaria; 2002.
8. Brasil. Lei no 11.346, de 15 de setembro de 2006. Cria o Sistema Nacional de Segurança Alimentar e Nutricional - SISAN com vistas em assegurar o direito humano à alimentação adequada e dá outras providências. Diário Oficial da União 2006; 18 set.

9. Organización de las Naciones Unidas para la Alimentación y la Agricultura. Declaración de Roma sobre la seguridad Alimentaria mundial y plan de acción de la cumbre mundial sobre la alimentación. Roma: Organización de las Naciones Unidas para la Alimentación y la Agricultura; 1996.

10. Holt-Giménez E, Patel R. Rebeliones alimentarias. Crisis y el hambre por la justicia. Barcelona: El Viejo Topo; 2010.

11. McMichael P. A food regime analysis of the 'world food crisis'. Agric Human Values 2009; 26:281-95.

12. Stevens C, Greenhill R, Kennan J, Devereux S. The WTO agreement on agriculture and food security. London: Commonwealth Secretariat; 2000.

13. McMichael P. A food regime genealogy. J Peasant Stud 2009; 36:139-69.

14. Hansen-Kuhn K. Making U.S. trade policy serve global food security goals. Sustainable Development Law \& Policy 2011; 11:9-38.

15. Mahony G. Foreign acquisition of agricultural land and food security: a cautionary note on public policy. Economic Papers 2012; 33:501-7.

16. Fernández-Wulff P. International trade and food security: can agrobiodiversity reconcile both? United Nations Peace and Progress 2013; 1:18-32. 
17. McMichael P. Global development and the corporate food regime. In: Buttel FH, McMichael P, editores. New directions in the sociology of global development. Bingley: Emerald Group Publishing; 2005. p. 269-99. (Research in Rural Sociology and Development, volume 11).

18. Paveliuc-Olariu C. Developing a global food security policy. Advances in Agriculture \& Botanics International Journal of the Bioflux Society 2013; 5:169-73.

19. Marsden T. Towards a real sustainable agri-food security and food policy: beyond the ecological fallacies? Polit Q 2012; 83:139-45.

20. Cook JT. Clinical implications of household food security: definitions, monitoring, and policy. Nutr Clin Care 2002; 5:152-67.

21. Brooks J, Matthews A. Agricultural trade and food security: choosing between trade and non-trade policy instruments. http://www.ictsdsymposium. org/wp-content/uploads/bsk-pdf-manager/31_ Brooks-Matthews-Trade-Policy-and-Food-Securi ty.pdf (accedido el 12/Ago/2013).

22. Flores M, Khwaja Y, White P. Food security in protracted crises: building more effective policy frameworks. Disasters 2005; 29 Suppl 1:S25-51.

23. Hefny MA. Changing behavior as a policy tool for enhancing food security. Water Policy 2012; 14:106-20.

24. Babu SC, Rhoe V. Agroforestry systems for food and nutrition security - potentials, pathwaysand policy research needs. Journal of Crop Production 2002; 6:177-92.

25. Kopainsky B, Tröger K, Derwisch S, Ulli-Beer S. Designing sustainable food security policies in Sub-Saharan African countries: how social dynamics over-ride utility evaluations for good and bad. Systems Research and Behavioral Science 2012; 29:575-89.

26. Rocha C. Developments in national policies for food and nutrition security in Brazil. Development Policy Review 2009; 27:51-66.

27. Burlandy L. Construction of the food and nutrition security policy in Brazil: strategies and challenges in the promotion of intersectorality at the federal government level. Ciênc Saúde Coletiva 2009; 14:851-60.

28. Paulillo L, Almeida L. Food safety and management of local public policy network: a comparative analysis of the cities of Campinas, Araraquara, and Catanduva. Gestão \& Produção 2011; 18:853-68.

29. Nova Scotia Nutrition Council; Atlantic Health Promotion Research Centre. A national environmental scan of strategies for influencing policy to build food security. Final report. Halifax: Atlantic Health Promotion Research Centre; 2004.

30. Organización de las Naciones Unidas para la Alimentación y la Agricultura. Políticas de seguridad alimentaria en los países de la Comunidad Andina. Oficina Regional de la FAO para América Latina y el Caribe. Santiago: Organización de las Naciones Unidas para la Alimentación y la Agricultura; 2005.
31. Organización de las Naciones Unidas para la Alimentación y la Agricultura. Informe de la $32^{\mathrm{a}}$ Conferencia Regional de la FAO para América Latina y el Caribe. Buenos Aires: Organización de las Naciones Unidas para la Alimentación y la Agricultura; 2012.

32. Organización de las Naciones Unidas para la Alimentación y la Agricultura. Comité de Seguridad Alimentaria Mundial. Marco estratégico mundial para la seguridad alimentaria y la nutrición. Primer proyecto. Roma: Organización de las Naciones Unidas para la Alimentación y la Agricultura; 2012.

33. La Vía Campesina. Food sovereignty and international trade. Bangalore: Japanada Loka; 2000.

34. Montagut X, Dogliotti F. Alimentos globalizados: soberanía alimentaria y comercio justo. Barcelona: Icaria; 2008.

35. Windfuhr M, Jonsén J. Food sovereignty. Towards democracy in localized food systems. Chippenham: ITDG Publishing; 2005.

36. Global Health Watch. Global Health Watch -3. http://www.ghwatch.org/ghw3.

37. Cook J. Addressing the "slow violence" of food insecurity. In: Scherrer C, Saha D, editores. The food crisis. Implications for labor. München: ICDD Rainer Hampp Verlag; 2013. p. 1-28.

38. Tomlinson I. Doubling food production to feed the 9 billion: a critical perspective on a keydiscourse of food security in the UK. J Rural Stud 2013; 29: 81-90.

39. Menezes F. Food sovereignty: a vital requirement for food security in the context of globalization. Development 2001; 44:30-3.

40. Beuchelt TD, Virchow D. Food sovereignty or the human right to adequate food: which concept serves better as international development policy for global hunger and poverty reduction? Agric Human Values 2012; 29:259-73.

41. Burmeister LL, Choi Y-J. Food sovereignty movement activism in South Korea: national policy impacts? Agric Human Values 2012; 29:247-58.

42. Dupraz CL, Postolle A. Food sovereignty and agricultural trade policy commitments: how much leeway do West African nations have? Food Policy 2013; 38:115-25.

43. Andrée P, Cobb M, Moussa L, Norgang E. Building unlikely alliances around food sovereignty in Canada. Studies in Political Economy 2011; 88:133-59.

44. Pimbert M. Towards food sovereignty. Gatekeeper 2009; 141:1-20.

45. Arthur PK. Food security and sovereignty in Africa: issues, policy challenges and opportunities. Harare: African Capacity Building Foundation; 2012. (ACBF Occasional Papers, 17).

46. Lee R. The politics of international agri-food policy: discourses of trade-oriented food security and food sovereignty. Env Polit 2012; 22:216-34. 
47. Pingali P, Alinovi L, Sutton J. Food security in complex emergencies: building food systems resilience. Tivoli: European Commission/Organización de las Naciones Unidas para la Alimentación y la Agricultura; 2005.

48. Organización de las Naciones Unidas para la Alimentación y la Agricultura. Informe de políticas no 2. Seguridad alimentaria. Roma: Organización de las Naciones Unidas para la Alimentación y la Agricultura; 2006.

49. McMichael P, Schneider M. Food security politics and the Millennium Development Goals. Third World Q 2011; 32:119-39.

50. Gross R, Schoeneberger H, Pfeifer H, HansJoachim A. Preuss: the four dimensions of food and nutrition security: definitions and concepts. http://www.nfpcsp.org/agridrupal/sites/default/ files/Four_Dimension_of_FS.pdf (accedido el 12/ Jan/2013)

51. Lang T. Food control or food democracy? Reengaging nutrition with society and the environment. Public Health Nutr 2005; 8:730-7.

52. Carrasco H, Tejada S. Soberanía alimentaria: la libertad de elegir para asegurar nuestra alimentación. Lima: Soluciones Prácticas; 2008.

53. Gerwin M, editor. Food and democracy: introduction to food sovereignty. Kraków: Polish Green Network; 2011.

54. Pinstrup-Andersen P. Agricultural research and policy for better health and nutrition in developing countries: a food systems approach. Agric Econ 2006; 37:187-98.

55. Coveney J. Why food policy is critical to public health. Crit Public Health 2003; 13:99-105.
56. Seed B. Food security in public health and other government programs in British Columbia, Canada: a policy analysis. London: Centre for Food Policy, School of Community and Health Sciences, City University; 2011.

57. Mikkonen J, Raphael D. Social determinants of health: the Canadian facts. Toronto: York University School of Health Policy and Management; 2010.

58. Seed B, Lang T, Caraher M, Ostry A. Integrating food security into public health and provincial government departments in British Columbia, Canada. Agric Human Values 2013: 30:457-70.

59. Hassanein N. Practicing food democracy: a pragmatic politics of transformation. J Rural Stud 2003; 19:77-86.

60. Friendly A. Towards food security policy for Canada's Social Housing Sector. [place unknown]: Canadian Policy Research Networks; 2008.

61. Bellows AC, Hamm MW. International effects on and inspiration for community food security policies and practices in the USA. Crit Public Health 2003; 13:107-23.

62. Caraher M, Coveney J. Public health nutrition and food policy. Public Health Nutr 2004; 7:591-8.

63. Lang T, Barling D, Caraher M. Food policy: integrating health, environment and society. Oxford: Oxford University Press; 2009.

64. Maceira D, Stechina M. Intervenciones de política alimentaria en 25 años de democracia en Argentina. Rev Cuba Salud Pública 2010; 37:44-60.

Recibido el 20/Ago/2014

Versión final presentada el 29/Ene/2015

Aprobado el 02/Mar/2015 